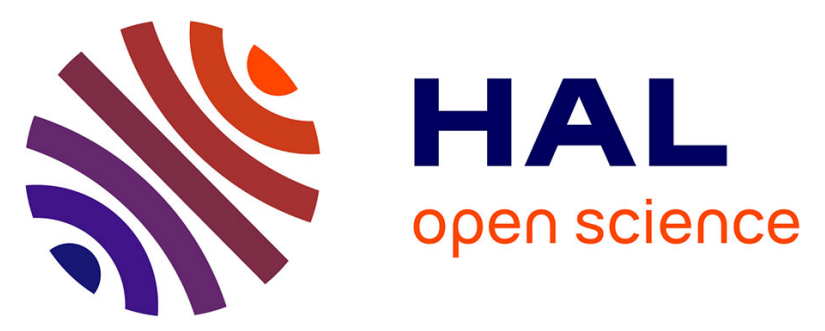

\title{
Simple Two-Body Cation-Water Interaction Potentials Derived from ab Initio Calculations. Comparison to Results Obtained with an Empirical Approach
}

\author{
X. Periole, David Allouche, J.-P. Daudey, Y.-H. Sanejouand
}

\section{- To cite this version:}

X. Periole, David Allouche, J.-P. Daudey, Y.-H. Sanejouand. Simple Two-Body Cation-Water Interaction Potentials Derived from ab Initio Calculations. Comparison to Results Obtained with an Empirical Approach. Journal of Physical Chemistry B, 1997, 101 (25), pp.5018-5025. 10.1021/jp9701855 . hal-02694213

\section{HAL Id: hal-02694213 \\ https://hal.inrae.fr/hal-02694213}

Submitted on 1 Jun 2020

HAL is a multi-disciplinary open access archive for the deposit and dissemination of scientific research documents, whether they are published or not. The documents may come from teaching and research institutions in France or abroad, or from public or private research centers.
L'archive ouverte pluridisciplinaire HAL, est destinée au dépôt et à la diffusion de documents scientifiques de niveau recherche, publiés ou non, émanant des établissements d'enseignement et de recherche français ou étrangers, des laboratoires publics ou privés. 


\title{
Simple Two-Body Cation-Water Interaction Potentials Derived from ab Initio Calculations. Comparison to Results Obtained with an Empirical Approach
}

\author{
X. Periole, D. Allouche, J.-P. Daudey, and Y.-H. Sanejouand* \\ Laboratoire de Physique Quantique, UMR 5626 of CNRS, IRSAMC, Université Paul Sabatier, \\ 118 route de Narbonne, 31062 Toulouse Cédex, France
}

Received: January 13, $1997^{\otimes}$

\begin{abstract}
Ab initio calculations were performed on $\mathrm{M}\left(\mathrm{H}_{2} \mathrm{O}\right)_{n}$ systems, $\mathrm{M}$ being $\mathrm{Li}^{+}, \mathrm{Na}^{+}, \mathrm{K}^{+}, \mathrm{Be}^{2+}, \mathrm{Mg}^{2+}$, or $\mathrm{Ca}^{2+}$, with $n=1,2,4$, or 6 . For the most hydrated systems, parameters for the effective Lennard-Jones interaction between the cation and the water molecules were determined, so as to reproduce $a b$ initio results. In order to compare our results to those obtained previously by J. Åqvist with a purely empirical approach, waterwater interactions were assumed to be given by the TIP3P model. Different forms for the effective two-body interaction potential were tested. The best fits of ab initio data were obtained with a smooth $r^{-7}$ repulsive and a classical $r^{-4}$ attractive term, in addition to standard Coulombic interactions. Though better fits were obtained for alkaline cations than for alkaline-earth ones, only $\mathrm{Be}^{2+}$ obviously requires a more complicated form of the potential energy function. The corresponding parameters were tested with molecular dynamics simulations of cations in water solutions and with hydration free energy difference calculations, using the thermodynamic perturbation approach. Radial distribution functions consistent with experimental data were obtained for all cations. Free energy differences are obviously much more challenging. The most accurately reproduced value is the difference between the hydration free energies of $\mathrm{Na}^{+}$and $\mathrm{K}^{+}$. This result is likely to be significant since effective interaction energies between $\mathrm{Na}^{+}$or $\mathrm{K}^{+}$and water molecules as obtained in Åvist's and in the present work are found to be very similar, despite the fact that the corresponding sets of parameters were determined with completely different approaches.
\end{abstract}

\section{Introduction}

Alkaline-earth and alkaline metal cations $\mathrm{Mg}^{2+}, \mathrm{Ca}^{2+}, \mathrm{Na}^{+}$, and $\mathrm{K}^{+}$play entirely different roles in biological systems. For instance, when the intracellular concentration of $\mathrm{Ca}^{2+}$ increases, large conformational changes occur in many proteins. In the case of muscle cells, this results in the activation of a protein kinase, which phosphorylates the glycogen phosphorylase enzyme, ultimately causing energy release from glycogen and muscle contraction. Interestingly, the high physiological intracellular concentration of $\mathrm{Mg}^{2+}$ does not interfere with such events $\left(\mathrm{Ca}^{2+}\right.$ is a key signaling molecule in eukaryotic cells, while $\mathrm{Mg}^{2+}$ is not). This is related to the fact that the specificity of proteins for $\mathrm{Ca}^{2+}$ can be fairly high. For instance, in the case of parvalbumin, the ratio of $\mathrm{Mg}^{2+}$ and $\mathrm{Ca}^{2+}$ binding affinity constants is $\approx 10^{4}$. $^{1}$

The most popular method presently available to study protien specificity at a molecular level is the free energy perturbation (FEP) method. ${ }^{2}$ With this method, the difference between the binding free energies of two ligands of a given protein can be computed. $^{3,4}$ In practice, accurate results are obtained only when the following conditions are met: (1) a high resolution tridimensional structure of one ligand-protein complex has to be known; (2) both ligands, and the conformation of the protein around them, must be similar enough so that the part of the configurational space in which the behavior of the two ligandprotein systems is different can be sampled during a molecular dynamics (MD) simulation at room temperature. To fulfill this condition it is usually necessary, even for very similar systems, to build nonphysical intermediaries between the two ligands, the free energy difference being calculated along the nonphysical

\footnotetext{
${ }^{\otimes}$ Abstract published in Advance ACS Abstracts, June 1, 1997.
}

path starting from one ligand and ending at the other one; ${ }^{2}(3)$ the interaction between the ligands and the protein must be well described. Moreover, in order to achieve at reasonable computational costs the large number of energy calculations required for an accurate sampling (typically, hundreds of thousands), this description has to be simple. Simplicity in the description also allows for parameter transferability. More generally, when the number of parameters in the potential energy function is kept low, a definite physical meaning can be assigned to each term of the function. In most programs currently devoted to MD studies of proteins, e.g., CHARMM,${ }^{5}$ the interaction between a cation and protein atoms is assumed to be a sum of LennardJones and electrostatic interactions between point charges. In order to save computer time, many-body terms are usually not included. Their effects are expected to be taken into account, on average, as effective electrostatic or Lennard-Jones interactions between atom pairs.

Recently, a new method for obtaining parameters for such effective interactions has been proposed by Åqvist, ${ }^{6}$ aiming at a correct description of the structural and dynamical behavior of a cation in a water solution. In this approach, while electrostatic interactions are supposed to be known from other sources and since Lennard-Jones interactions between the cation and hydrogen atoms are neglected, only two parameters have to be determined, namely, those of the Lennard-Jones interaction between the cation and the oxygen of water molecules. These parameters are chosen so that values close to experimental ones are obtained from MD and FEP simulations for two quantities: the average distance between the cation and the oxygen atoms in its first hydration shell, and the absolute free energy of solvation of the cation. 
TABLE 1: $\mathrm{Ca}^{2+}\left(\mathrm{H}_{2} \mathrm{O}\right)_{n}$. Optimized Geometries

\begin{tabular}{ccccl}
\hline$n$ & $r_{\text {opt }}(\AA)$ & $E_{\mathrm{Q}_{\text {int }}}^{n}(\mathrm{kcal} / \mathrm{mol})$ & $E_{\mathrm{Q}_{\text {tot }}}^{n}(\mathrm{kcal} / \mathrm{mol})$ & source \\
\hline 1 & 2.28 & -54.8 & -54.8 & this work \\
1 & 2.28 & -55.0 & -55.0 & 16 \\
1 & 2.30 & -53.7 & -53.7 & 18 \\
1 & 2.28 & -55.6 & -55.6 & 17 \\
1 & 2.26 & -53.3 & -53.3 & 24 \\
1 & 2.34 & -56.1 & -56.1 & 10 \\
1 & 2.28 & -56.5 & -56.5 & 25 \\
2 & 2.33 & -48.7 & -103.3 & this work \\
2 & 2.31 & -48.9 & & 16 \\
2 & 2.34 & & -102.0 & 18 \\
2 & 2.27 & -51.8 & -113.6 & 24 \\
2 & 2.36 & -51.6 & & 10 \\
2 & 2.31 & & -107.0 & 25 \\
6 & 2.43 & -31.2 & -246.5 & this work \\
6 & 2.44 & & -244.0 & 18 \\
6 & 2.44 & -31.8 & & 10 \\
6 & 2.43 & & -253.0 & 25
\end{tabular}

TABLE 2: $\operatorname{Mg}^{2+}\left(\mathrm{H}_{2} \mathrm{O}\right)_{n}$. Optimized Geometries

\begin{tabular}{ccccl}
\hline$n$ & $r_{\mathrm{opt}}(\AA)$ & $E_{\mathrm{Q}_{\text {int }}}^{n}(\mathrm{kcal} / \mathrm{mol})$ & $E_{\mathrm{Q}_{\text {tot }}}^{n}(\mathrm{kcal} / \mathrm{mol})$ & \multicolumn{1}{c}{ source } \\
\hline 1 & 1.92 & -81.2 & -81.2 & this work \\
1 & 1.92 & -81.9 & -81.9 & 16 \\
1 & 1.94 & -78.8 & -78.8 & 18 \\
1 & 1.99 & -73.8 & -73.8 & 24 \\
1 & 1.95 & -85.8 & -85.8 & 19 \\
2 & 1.93 & -74.2 & -155.2 & this work \\
2 & 1.93 & -73.8 & -149.4 & 16 \\
2 & 1.96 & & -153.2 & 24 \\
2 & 1.98 & -71.6 & & 19 \\
2 & 1.96 & -77.8 & -326.0 & this work \\
6 & 2.10 & -36.0 & -313.1 & 18 \\
6 & 2.11 & & & 19 \\
6 & 2.10 & -34.0 & &
\end{tabular}

TABLE 3: $\mathrm{Be}^{2+}\left(\mathrm{H}_{2} \mathrm{O}\right)_{n}$. Optimized Geometries

\begin{tabular}{ccccl}
\hline$n$ & $r_{\text {opt }}(\AA)$ & $E_{\mathrm{Q}_{\text {int }}}^{n}(\mathrm{kcal} / \mathrm{mol})$ & $E_{\mathrm{Q}_{\text {tot }}}^{n}(\mathrm{kcal} / \mathrm{mol})$ & \multicolumn{1}{c}{ source } \\
\hline 1 & 1.51 & -140.6 & -140.6 & this work \\
1 & 1.51 & -149.7 & -149.7 & 19 \\
4 & 1.66 & -65.6 & -386.3 & this work \\
4 & 1.65 & -56.0 & & 19
\end{tabular}

The purpose of our study is to check the main hypothesis Åqvist's work is based on. At a more general level, the question of how well a quantum mechanical interaction energy between a water molecule and a cation can be approximated by simple effective two-body interactions will be addressed, in the case of systems of increasing complexity, $\mathrm{M}\left(\mathrm{H}_{2} \mathrm{O}\right)_{n}$, with $n$ ranging from 1 up to $6, \mathrm{M}$ being $\mathrm{Ca}^{2+}, \mathrm{Mg}^{2+}, \mathrm{Na}^{+}, \mathrm{K}^{+}$, as well as another alkaline-earth metal cation, $\mathrm{Be}^{2+}$, and another alkaline one, $\mathrm{Li}^{+}$(the two later cations have also important, though nonphysiological, biological effects).

The main principle of our work is to study such an interaction in a "realistic" water environment. To do so, in the largest systems considered, the first hydration shell of the cation was filled according to experimental data. However, while it is quite clear that there are respectively 4 and 6 water molecules in the first hydration shell of $\mathrm{Be}^{2+}$ and $\mathrm{Mg}^{2+}$, experimental data related to other cations are less conclusive. ${ }^{7}$ For instance, values ranging from 6 to 10 were found in X-ray and neutron diffraction experiments for $\mathrm{Ca}^{2+}{ }^{7}$ In Monte Carlo and molecular dynamics studies, values ranging from $7^{8}$ to $9^{9}$ were observed, while it was shown with $a b$ initio calculations that a $\mathrm{Ca}^{2+}$ with 8 water molecules in its first hydration shell is slightly more stable than with $9 .{ }^{10}$ In the present study, the $n=6$ case was assumed to be a representative one for all cations, except for $\mathrm{Be}^{2+}$. The $n$ $=1$ and $n=2$ cases were also considered, both in order to
TABLE 4: $\mathrm{K}^{+}\left(\mathrm{H}_{2} \mathrm{O}\right)_{n}$. Optimized Geometries

\begin{tabular}{ccccl}
\hline$n$ & $r_{\text {opt }}(\AA)$ & $E_{\mathrm{Q}_{\text {int }}}^{n}(\mathrm{kcal} / \mathrm{mol})$ & $E_{\mathrm{Q}_{\text {tot }}}^{n}(\mathrm{kcal} / \mathrm{mol})$ & \multicolumn{1}{c}{ source } \\
\hline 1 & 2.69 & -17.5 & -17.5 & this work \\
1 & 2.66 & -18.6 & -18.6 & 26,27 \\
1 & 2.60 & -23.0 & -23.0 & 20 \\
6 & 2.81 & -9.6 & -79.4 & this work \\
6 & 2.89 & -7.8 & -82.1 & 26,27
\end{tabular}

TABLE 5: $\mathrm{Na}^{+}\left(\mathrm{H}_{2} \mathrm{O}\right)_{n}$. Optimized Geometries

\begin{tabular}{ccccl}
\hline$n$ & $r_{\mathrm{opt}}(\AA)$ & $E_{\mathrm{Q}_{\text {int }}}^{n}(\mathrm{kcal} / \mathrm{mol})$ & $E_{\mathrm{Q}_{\text {tot }}}^{n}(\mathrm{kcal} / \mathrm{mol})$ & \multicolumn{1}{c}{ source } \\
\hline 1 & 2.23 & -25.8 & -25.8 & this work \\
1 & 2.23 & -25.1 & -25.1 & 26 \\
1 & 2.21 & -28.7 & -28.7 & 20 \\
6 & 2.40 & -11.5 & -106.5 & this work \\
6 & 2.42 & -10.3 & -100.2 & 26
\end{tabular}

TABLE 6: $\mathrm{Li}^{+}\left(\mathrm{H}_{2} \mathrm{O}\right)_{n}$. Optimized Geometries

\begin{tabular}{ccccl}
\hline$n$ & $r_{\text {opt }}(\AA)$ & $E_{\mathrm{Q}_{\text {int }}}^{n}(\mathrm{kcal} / \mathrm{mol})$ & $E_{\mathrm{Q}_{\text {tot }}}^{n}(\mathrm{kcal} / \mathrm{mol})$ & \multicolumn{1}{c}{ source } \\
\hline 1 & 1.84 & -36.5 & -36.5 & this work \\
1 & 1.82 & -35.6 & -35.6 & 26 \\
1 & 1.85 & -39.7 & -39.7 & 20 \\
6 & 2.14 & -11.4 & -125.8 & this work \\
6 & & & -119.7 & 26
\end{tabular}

compare the results of our ab initio calculations with an extended set of results obtained by other groups, and to emphasize the need of considering rather large systems when studying the interactions of a cation in polar densed environments.

In the following, parameters extracted from ab initio calculations will be compared to those obtained by Åqvist with his empirical approach. Results of free energy differences calculations performed with these parameters will be compared to experimental data.

\section{Methods}

Ab Initio Calculations. For the calculation of molecular interactions between ionic species, the crucial aspect is the correct evaluation of the electrostatic and charge contributions, which are the dominant parts of the interaction. Since all these contributions are readily included at the Hartree-Fock (HF) level, we decided to use this level for the bulk of our calculations. As a matter of fact, in this specific case, the basis set superposition (BSSE) correction (which decreases the interaction energy) is largely compensated by the neglect of post-Hartree-Fock contributions. This is illustrated in Tables 1-6 where we compare our results for small clusters with more refined calculations in which both BSSE and correlation are taken into account. We also have performed some additional test calculations in the case of $\mathrm{Ca}^{2+}\left(\mathrm{H}_{2} \mathrm{O}\right)_{6}$. The BSSE correction decreases the interaction energy at equilibrium by $\approx 6.0 \%$ while, when some correlation energy is included, at the MP2 level, it is increased by $\approx 10.0 \%$. The overall precision obtained seems to be sufficient for our purpose which implies a very detailed analysis of the potential energy surface and a large number of calculations.

The atomic basis sets have been extracted from the TURBOMOLE library, ${ }^{11}$ except for $\mathrm{Ca}^{2+}$, for which an effective core potential of 10 electrons for $\mathrm{Ca}^{2+}$ and the corresponding basis set was derived in previous works. ${ }^{12}$ Since polarization effects are mostly important for the oxygen atom, we chose a TZP (triple zeta + polarization) basis including two $\mathrm{d}$ functions. Thus, basis set sizes are as follows:

$\mathrm{Ca}^{2+} \quad(6 \mathrm{~s} 8 \mathrm{p} 5 \mathrm{~d}) /[4 \mathrm{~s} 6 \mathrm{p} 2 \mathrm{~d}]$ 


$$
\begin{array}{ll}
\mathrm{Mg}^{2+} & (11 \mathrm{~s} 7 \mathrm{p} 1 \mathrm{~d}) /[6 \mathrm{~s} 3 \mathrm{p} 1 \mathrm{~d}] \\
\mathrm{Be}^{2+} & (9 \mathrm{~s} 2 \mathrm{p}) /[5 \mathrm{~s} 2 \mathrm{p}] \\
\mathrm{K}^{+} & (14 \mathrm{~s} 9 \mathrm{p} 1 \mathrm{~d}) /[9 \mathrm{~s} 5 \mathrm{p} 1 \mathrm{~d}] \\
\mathrm{Na}^{+} & (11 \mathrm{~s} 7 \mathrm{p} 2 \mathrm{~d}) /[6 \mathrm{~s} 3 \mathrm{p} 2 \mathrm{~d}] \\
\mathrm{Li}^{+} & (9 \mathrm{~s} 2 \mathrm{p}) /[4 \mathrm{~s} 2 \mathrm{p}] \\
\mathrm{O} & (9 \mathrm{~s} 5 \mathrm{p} 2 \mathrm{~d}) /[5 \mathrm{~s} 3 \mathrm{p} 2 \mathrm{~d}] \\
\mathrm{H} & (4 \mathrm{~s} 2 \mathrm{p}) /[2 \mathrm{~s} 2 \mathrm{p}]
\end{array}
$$

Extensive $a b$ initio calculations were performed on the following systems: $\mathrm{Ca}^{2+}\left(\mathrm{H}_{2} \mathrm{O}\right)_{n}$ with $n=1,2$, and $6, \mathrm{Mg}^{2+}$. $\left(\mathrm{H}_{2} \mathrm{O}\right)_{n}$ with $n=1,2$, and $6, \mathrm{Be}^{2+}\left(\mathrm{H}_{2} \mathrm{O}\right)_{n}$ with $n=1$ and 4 , $\mathrm{K}^{+}\left(\mathrm{H}_{2} \mathrm{O}\right)_{n}, \mathrm{Na}^{+}\left(\mathrm{H}_{2} \mathrm{O}\right)_{n}$, and $\mathrm{Li}^{+}\left(\mathrm{H}_{2} \mathrm{O}\right)_{n}$ with $n=1$ and 6 . Water clusters around a given cation were built with the following symmetry: $C_{2 v}(n=1), D_{2 d}(n=2$ and 4$)$ and $T_{h}(n=6)$. In each case, a geometry optimization was performed. Then, one water molecule was translated away or toward the cation, the interaction energy of this water molecule with the cation and the other $n-1$ water molecules, $E_{\mathrm{Q}_{\mathrm{int}}}^{n}(r)$ being determined according to

$$
E_{\mathrm{Q}_{\text {int }}}^{n}(r)=E_{\mathrm{Q}}^{n}(r)-E_{\mathrm{Q}}^{n-1}-E_{\mathrm{Q}}^{\mathrm{w}}
$$

where $r$ is the distance between the cation and the oxygen atom of the translated water molecule, $E_{\mathrm{Q}}^{\mathrm{w}}$ is the energy of this water molecule, $E_{\mathrm{Q}}^{n}(r)$ is the energy of the whole system, and $E_{\mathrm{Q}}^{n-1}$ is the energy of the system when the translated molecule is removed. In order to compare our results with previous ones, the total interaction energy of the system at equilibrium, $E_{\mathrm{Q}_{\text {tot }}}^{n}$, was also calculated, according to

$$
E_{\mathrm{Q}_{\mathrm{tot}}}^{n}=E_{\mathrm{Q}}^{n}\left(r_{\mathrm{opt}}\right)-E_{\mathrm{Q}}^{\mathrm{c}}-n E_{\mathrm{Q}}^{\mathrm{w}}
$$

where $E_{\mathrm{Q}}^{\mathrm{c}}$ is the energy of the cation.

Effective Two-Body Interaction Energy Parameters. For each system, two or three parameters were determined, $A_{n}, B_{n}$, and $C_{n}$, with a least-squares-fit procedure, so at to minimize the mean square difference between $E_{\mathrm{Q}_{i n t}}^{n}(r)$, the above quantum mechanical interaction energy, and the following classical one, either a " $c-d$ Lennard-Jones" form:

$$
E_{\mathrm{LJ}}^{n}(r)=\frac{A_{n}}{r^{c}}-\frac{B_{n}}{r^{d}}+\sum_{i}^{n-1}\left\{\frac{A_{\mathrm{oo}}}{R_{i}^{12}}-\frac{B_{\mathrm{oo}}}{R_{i}^{6}}\right\}+E_{\mathrm{elec}}^{n}(r)
$$

or a " $d$-Buckingham" one:

$$
E_{\text {Buck }}^{n}(r)=A_{n} \exp \left(-C_{n} r\right)-\frac{B_{n}}{r^{d}}+\sum_{i}^{n-1}\left\{\frac{A_{\mathrm{oo}}}{R_{i}^{12}}-\frac{B_{\mathrm{oo}}}{R_{i}^{6}}\right\}+E_{\text {elec }}^{n}(r)
$$

where $R_{i}$ is the distance between the oxygen of the translated water molecule and the oxygen of one among the $n-1$ other water molecules. $A_{\mathrm{oo}}$ and $B_{\mathrm{oo}}$ are the Lennard-Jones parameters for the TIP3P water model, ${ }^{13}$ which is widely used by CHARMM users. $E_{\text {elec }}^{n}(r)$ is the Coulombic energy of interaction between the translated water molecule and the rest of the system. Unless specified otherwise, electrostatic interactions between atoms of the translated water molecule and atoms of the rest of the system were calculated with standard charges: $q_{\mathrm{c}}=+1$ or $+2, q_{\mathrm{O}}=-0.834$, and $q_{\mathrm{H}}=+0.417$. As in Åquist's work, Lennard-Jones interactions in which hydrogen atoms are involved were neglected. Note that a 12-6 Lennard-Jones form was used by Åqvist in his MD and FEP calculations of cations in a water solution. ${ }^{6}$ In particular, the parameters of the water model are the same (Åqvist performed calculations either with the TIP3P or the SPC water model. With both models, a given set of $\left\{A_{n}, B_{n}\right\}$ lead to very similar values for solvation free energies). All these features will allow direct comparisons between the potential energy functions obtained in the present study with the $a b$ initio approach described above and by Aqvist with his empirical approach.

Molecular Dynamics and Free Energy Difference Calculations. Radial distribution functions (RDF) of water oxygens around cations were computed from the last 20 ps of 30 ps MD simulations performed at $300 \mathrm{~K}$ with a modified version of the CHARMM-22 program package. ${ }^{5}$ Simulation parameters are standard ones. In particular, bond lengths were constrained with the SHAKE algorithm, ${ }^{14}$ a 2 fs integration time step was used, and nonbonded interactions were calculated with a $14 \AA$ cutoff and a SHIFT truncation procedure for electrostatics. ${ }^{5}$ All Lennard-Jones interactions between the cation and water molecules were taken into account.

The solution model is as follows: the cation is held fixed at the center of a $15 \AA$ sphere of TIP3P water molecules. Water molecules lying more than $11 \AA$ away from the cation are also held fixed, as well as water oxygens lying more than $9 \AA$ away. Thus, water molecules in the three first hydration shells of the cation are free to move within a $9 \AA$ radius sphere surrounded by a $2 \AA$ soft boundary, in which water molecules are only free to rotate. This model was designed in order to perform free energy perturbation calculations both in water and in a protein environment. Since it is different from the one used by Aqvist, it was checked that results obtained with both models are similar. To do so, MD and free energy difference calculations were performed with our water solution model and the parameters and potential energy functions used by Åqvist in his study, both with the SPC and the TIP3P water models (data not shown).

Differences of hydration free energies were computed with the thermodynamic perturbation method. The principle of such calculations is as follows: first, several MD simulations are performed during which a cation in a water solution (state "a" of the system) is transformed into another (state " $b$ "), by varying a $\lambda$ parameter in the potential energy function of interaction of the cation with the water molecules. Then, the free energy difference between states $a$ and $b$ is obtained from ${ }^{15}$

$$
\Delta G_{\mathrm{ab}}=-k_{\mathrm{B}} T \sum_{i} \ln \left(\left.\exp \left[-\frac{E\left(\lambda_{i}+\Delta \lambda\right)-E\left(\lambda_{i}\right)}{k_{\mathrm{B}} T}\right]\right|_{\lambda_{i}}\right.
$$

where $k_{\mathrm{B}}$ is the Boltzmann constant, $T$ is the absolute temperature, and the brackets indicate that an ensemble average is calculated for each $\lambda_{i}$ value. Note that there is no approximation involved in this equation. From a practical point of view, for each free energy difference calculation, $10 \mathrm{MD}$ simulations at room temperature were performed, each with a given value of $\lambda_{i}$. In each simulation, a 5 ps equilibration period was followed by a 10 ps trajectory, the coordinates obtained at the end of a given simulation being the starting point of the next simulation, performed with a different value of $\lambda_{i}$, namely, $\lambda_{i}+\Delta \lambda$.

\section{Results and Discussion}

Optimized Geometries. In Tables 1-6, optimized geometries obtained at the HF level are compared to geometries obtained by other groups for the systems considered in the present study. Our results are in good agreement with previous 
works. For example, for $n=1$, the $\mathrm{Ca}-\mathrm{O}$ distance $(2.28 \AA$; see Table 1) is found to be within $0.01 \AA$ of the distances obtained by Bauschlicher et al. and Kaupp et al., despite the fact that these authors used a more extended set of basis functions than ours, including diffuse ones. ${ }^{16,17}$ In the $n=2$ case, $\mathrm{Ca}-\mathrm{O}$ distances are found to be slightly longer $(2.33 \AA$ ) than those obtained by Bauschlicher et al. $(2.31 \AA)$. However, this group studied a system in a $C_{2}$ geometry, while it was shown that with a system in a $D_{2 d}$ geometry the $\mathrm{Ca}-\mathrm{O}$ distance increases significantly. ${ }^{18}$

For $\mathrm{Mg}^{2+}\left(\mathrm{H}_{2} \mathrm{O}\right)_{n}$, in both $n=1$ and $n=2$ cases, $\mathrm{Mg}-\mathrm{O}$ distances are the same as in Bauschlicher et al. study: 1.92 and $1.93 \AA$, respectively (see Table 2 ). In other works, these distances are slightly longer than ours. For $\mathrm{Be}^{2+}\left(\mathrm{H}_{2} \mathrm{O}\right)_{n}$ (see Table 3), our geometrical results are in complete agreement with those obtained by Bock et al. ${ }^{19}$

Note that, in all studies, when $n$ increases, the cation-oxygen distance at equilibrium also increases. This is a trivial effect mostly due to water-water repulsion. There is also a relationship between the differences of cation-oxygen distances at equilibrium found in the present work and in the other works considered, and the corresponding differences of interaction energies. For instance, in the case of $\mathrm{Ca}^{2+}\left(\mathrm{H}_{2} \mathrm{O}\right)_{n}$ and $\mathrm{Mg}^{2+}$. $\left(\mathrm{H}_{2} \mathrm{O}\right)_{n}$, when there is a relative energy difference of more than $5 \%$, the cation-oxygen distances differ by more than $0.05 \AA$. If this trend is left apart, that is, if energies are compared for a given geometry, energy differences are expected to arise from the level of accuracy of the calculations. Nevertheless, as mentioned before, several sources of error may cancel out each other. For instance, for $\mathrm{Ca}^{2+}\left(\mathrm{H}_{2} \mathrm{O}\right)_{n}$, the Glendennig and Feller results and ours are similar both as far as geometries and energies are concerned, though the former include Counterpoise and MP2 corrections ${ }^{18}$ while ours do not. The effect of these corrections was checked in the case of $\mathrm{Ca}^{2+}\left(\mathrm{H}_{2} \mathrm{O}\right)_{6}$, whose total interaction energy decreases by $\approx 6.0 \%$ when BSSE corrections are taken into account while it increases by $\approx 10.0 \%$ when the calculations are performed at the MP2 level (data not shown). Since on the other hand it is clear from Tables 1-6 that interaction energies may vary from study to study by up to $10 \%$ (especially for $n=1$ cases), we found it not necessary to perform heavy calculations at the present stage of our work.

Optimization of the Form of the Classical Potential Energy Function. In Figure 1, the $a b$ initio interaction energy between $\mathrm{Ca}^{2+}$ and one water molecule is given, as a function of $r$, together with the best fits of these data obtained with, respectively, a 12-6 Lennard-Jones, a 7-4 Lennard-Jones, or a 6-Buckingham form of the classical interaction energy. It is clear that the 12-6 Lennard-Jones form, which was assumed by Åquist in his work, is not the best possible choice. In order to describe accurately the effective interaction energy between the cation and the water molecule, the 7-4 Lennard-Jones or the 6-Buckingham form performs obviously much better.

Other forms were also tested. A set of representative tests is given in Table 7 . With the $c-d$ Lennard-Jones forms, the switch from $d=6$ to $d=4$ improves the quality of the classical description, as measured by the mean-square difference (msd) between the quantum mechanical and the classical interaction energy. This is an expected result, since a $r^{-4}$ attractive term is the usual form for the interaction between a charge and an induced dipole. More unexpected is the extent of the improvement of the quality of the description with the Lennard-Jones form, when $c$ drops from $c=12$ to $c=7$. Note that the usual $r^{-12}$ repulsive term has no known physical meaning. Indeed, other values for $c$ have been proposed. For instance, Roux and Karplus used a $r^{-8}$ repulsive term in their description of alkaline

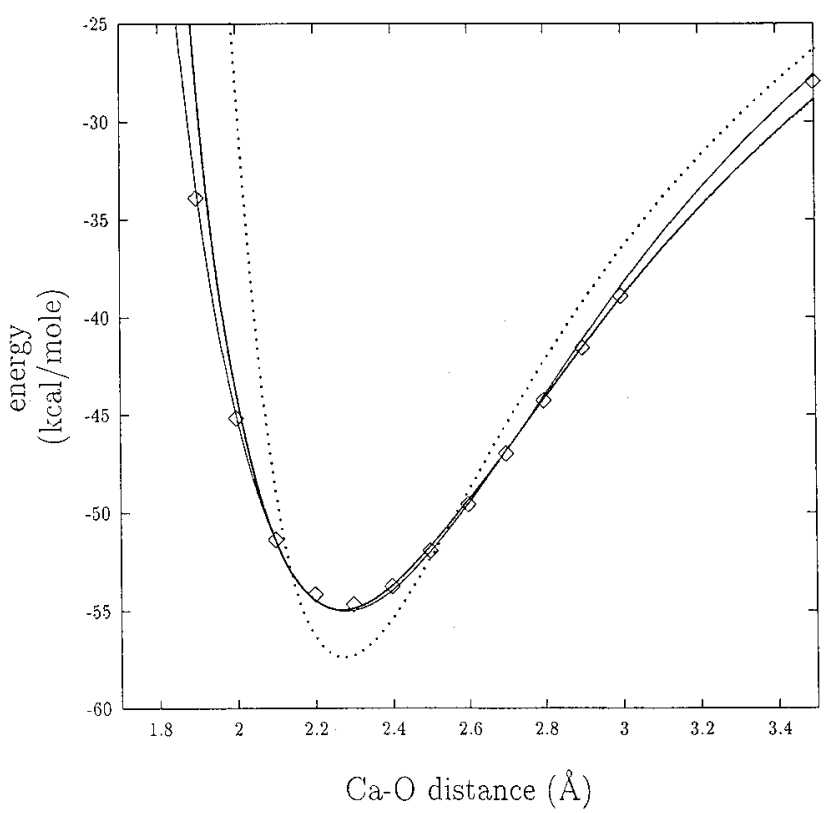

Figure 1. Interaction energy between $\mathrm{Ca}^{2+}$ and a water molecule, as a function of the distance between $\mathrm{Ca}^{2+}$ and the oxygen of the water molecule. $\diamond:$ ab initio results. These data were fitted with a potential energy function including a Coulombic term and, respectively, a standard 12-6 Lennard-Jones term (dotted line), a 7-4 Lennard-Jones term (plain line) and a 6-Buckingham term (continuous line).

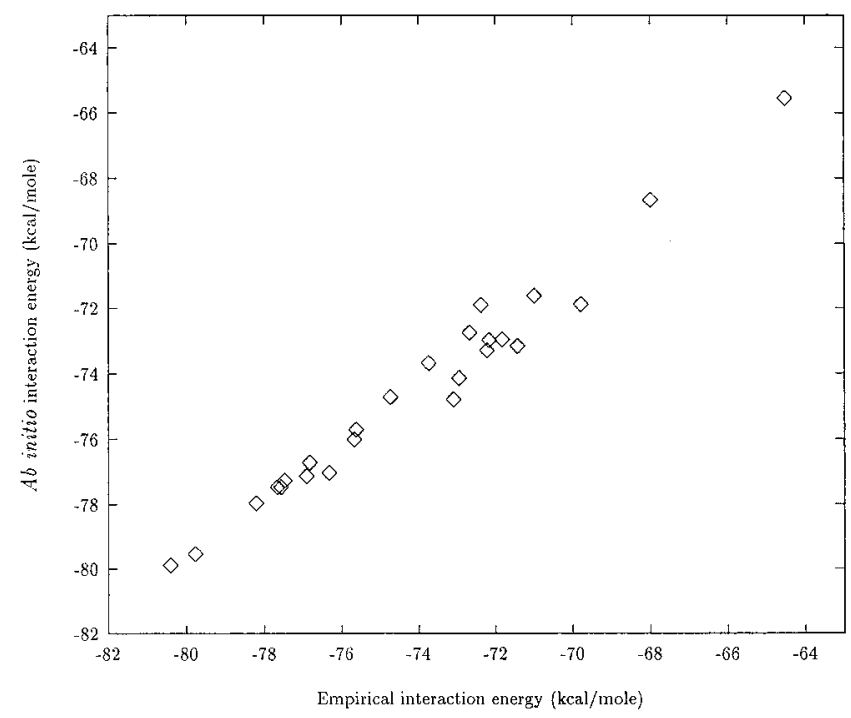

Figure 2. Comparison between ab initio and effective 7-4 LennardJones interaction energies for 25 nonsymmetric $\mathrm{Mg}^{2+}\left(\mathrm{H}_{2} \mathrm{O}\right)$ configurations.

TABLE 7: $\operatorname{Mg}^{2+}\left(\mathrm{H}_{2} \mathrm{O}\right)$. Fit of $a b$ Initio Data with Different $c-d$ Lennard-Jones Functions

\begin{tabular}{rrc}
\hline$c$ & $d$ & $\mathrm{msd}(\mathrm{kcal} / \mathrm{mol})^{2}$ \\
\hline 12 & 6 & 10.14 \\
10 & 6 & 7.06 \\
9 & 6 & 5.48 \\
8 & 6 & 3.93 \\
7 & 6 & 2.53 \\
12 & 4 & 4.47 \\
10 & 4 & 2.66 \\
9 & 4 & 1.94 \\
8 & 4 & 1.48 \\
7 & 4 & 1.45 \\
6 & 4 & 2.11
\end{tabular}

cation-carbonyl oxygen interaction, ${ }^{20}$ as well as Kowall et al. in their description of lanthanide ions-water oxygen interaction. $^{21}$ These later authors underline the fact that a smoother 
TABLE 8: $\quad \mathrm{M}^{+}\left(\mathrm{H}_{2} \mathrm{O}\right)_{n}$. Fit of ab Initio Data with Lennard-Jones Functions

\begin{tabular}{lcccc}
\hline cation & $n$ & $\begin{array}{c}\text { 7-4 Lennard-Jones } \\
\text { msd (kcal/mol) }\end{array}$ & $\begin{array}{c}\text { 6-Buckingham } \\
\text { msd (kcal/mol) }\end{array}$ & $\begin{array}{c}\text { 4-Buckingham } \\
\text { msd (kcal/mol) }\end{array}$ \\
\hline $\mathrm{Li}^{+}$ & 1 & 0.44 & 0.09 & 0.14 \\
$\mathrm{Na}^{+}$ & 1 & 0.14 & 0.03 & 0.06 \\
$\mathrm{~K}^{+}$ & 1 & 0.20 & 0.05 & 0.05 \\
$\mathrm{Be}^{2+}$ & 1 & 39.27 & 44.50 & 3.44 \\
$\mathrm{Mg}^{2+}$ & 1 & 1.45 & 0.54 & 0.93 \\
$\mathrm{Ca}^{2+}$ & 1 & 0.50 & 0.10 & 0.27 \\
$\mathrm{Li}^{+}$ & 6 & 0.07 & 0.03 & 0.01 \\
$\mathrm{Na}^{+}$ & 6 & 0.07 & 0.02 & 0.02 \\
$\mathrm{~K}^{+}$ & 6 & 0.09 & 0.02 & 0.02 \\
$\mathrm{Be}^{2+}$ & 4 & 1.75 & 1.27 & 1.45 \\
$\mathrm{Mg}^{2+}$ & 6 & 0.70 & 0.59 & 0.40 \\
$\mathrm{Ca}^{2+}$ & 6 & 0.45 & 0.43 & 0.20
\end{tabular}

Lennard-Jones repulsion results in a broadening of the first maximum of the radial distribution function of water oxygens around the cation and improves the agreement with neutron diffraction data. However, note that in the present work Lennard-Jones or Buckingham forms are here to complement the description of the interaction between the cation and the water molecules. In particular, the repulsive and attractive parts of the classical potential energy function are expected to correct errors introduced by the rough description of the electrostatic interactions in our systems.

Results given in Table 7 were found to be quite general: for all systems studied, the 7-4 Lennard-Jones performs much better than the usual 12-6 one (data not shown) while, as shown in Table 8, the 4- and 6-Buckingham forms were found to perform even better, the former being slightly more accurate when larger systems (i.e., more realistic ones) are considered. These later results are also expected since, as the number of parameters in the function used increases, better fits of the datas are usually obtained.

Other trends are noticeable in Table 8. First, better fits were obtained for alkaline cations than for alkaline-earth ones. Moreover, in most cases the quality of the fit increases as the radius of the cation increases, the worst fits being obtained with $\mathrm{Be}^{2+}$. In other words, as the strength of the water-cation interaction decreases, its description by a Buckingham form becomes more and more relevant.

Additional terms would be required in order to describe more accurately alkaline-earth cation-water oxygen interactions. Such terms would probably help to take into account physical effects like charge transfer, etc.

Second, better fits are in most cases obtained when the number of water molecules around the cation is large. For instance, for $\mathrm{Be}^{2+}\left(\mathrm{H}_{2} \mathrm{O}\right)$, msd values lie within 3.4-44.5, while for $\mathrm{Be}^{2+}\left(\mathrm{H}_{2} \mathrm{O}\right)_{4}$, they lie within 1.3-1.8. This trend is likely to be a consequence of the fact that cation-oxygen distances are larger when the first hydration shell of the cation is filled.

In all cases considered, the strength of the effective interaction between a cation and a single water molecule was found to decrease as the number of water molecules in the system used for parameter determination increases (see Figures 3-5 for a comparison between $n=1$ and $n=6$ cases). Parameter values obtained for the 4-Buckingham form are given in Table 9. The main feature is that low msd values shown in Table 8 were in many instances reached with negative $B_{n}$ values, the $r^{-4}$ term standing here to complement the description of the repulsive interaction between the cation and the water molecule. Such a feature is also observed with the 6-Buckingham form (data not shown). Nevertheless, one interesting result is the fact that $C_{n}$ values are found to lie within a rather narrow range: from 3.6 to 4.9. This suggests that a value near 4 may have some

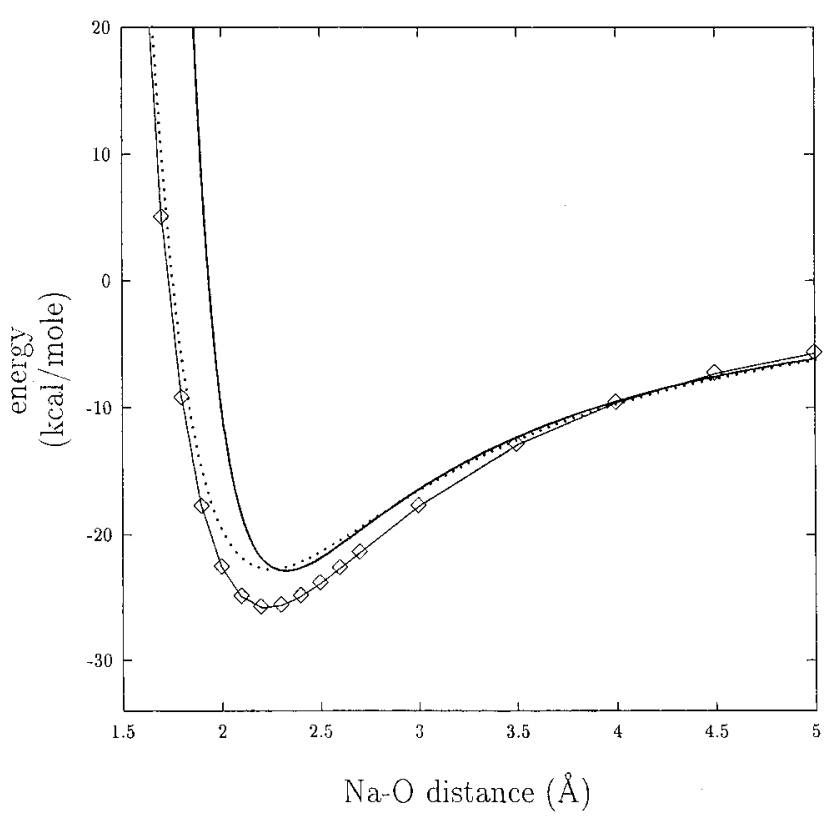

Figure 3. Interaction energy between $\mathrm{Na}^{+}$and a water molecule, as a function of the distance between $\mathrm{Na}^{+}$and the oxygen of the water molecule. $\diamond$ and continuous line: $a b$ initio results. Plain line: effective interaction energy, as obtained with Aqvist's empirical approach. Dotted line: effective interaction energy, as obtained with the present approach, $\mathrm{Na}^{+}$being in the field of five other water molecules.

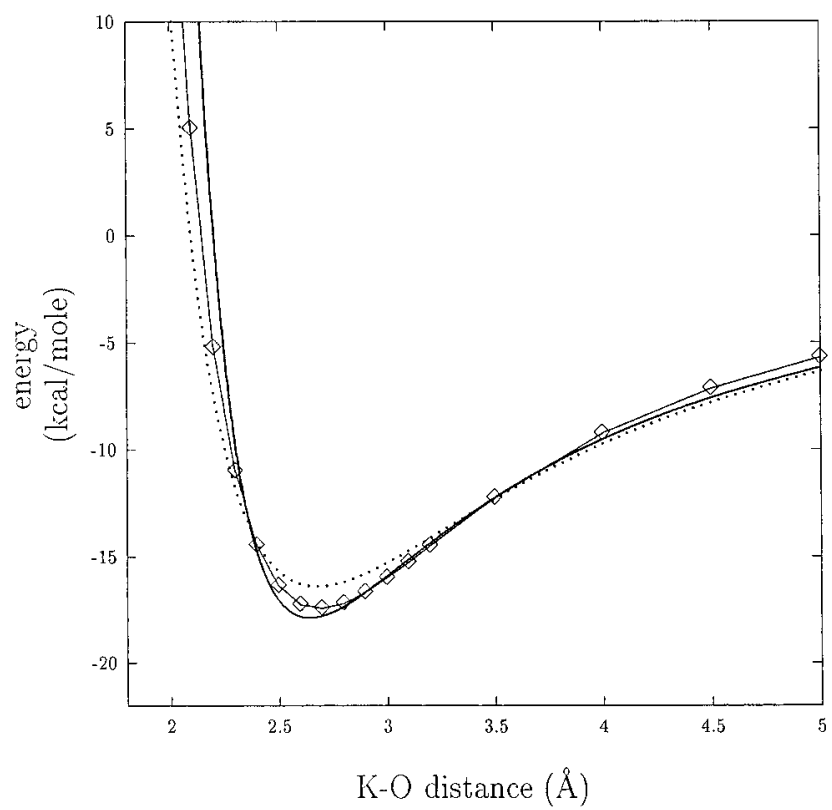

Figure 4. Interaction energy between $\mathrm{K}^{+}$and a water molecule, as a function of the distance between $\mathrm{K}^{+}$and the oxygen of the water molecule. $\diamond$ and continuous line: $a b$ initio results. Plain line: effective interaction energy, as obtained with Åqvist's empirical approach. Dotted line: effective interaction energy, as obtained with the present approach, $\mathrm{K}^{+}$being in the field of five other water molecules.

physical meaning. Other fits performed with a less polar water model $\left(q_{\mathrm{O}}=-0.7\right)$ confirm the robust character of this result (data not shown). Such fits were found to be very accurate. Moreover, they were reached with positive $A_{n}$ and $B_{n}$ values. This is likely to be meaningful since the dipolar moment of the modified water model was set to the value obtained at the HF level for a single water molecule, namely, $1.99 \mathrm{D}$-while it is $2.35 \mathrm{D}$ for the TIP3P model and $1.86 \mathrm{D}$ experimentally (in the gas phase). However, modifying the charges in the water model is a way to introduce a fourth parameter in the function used 


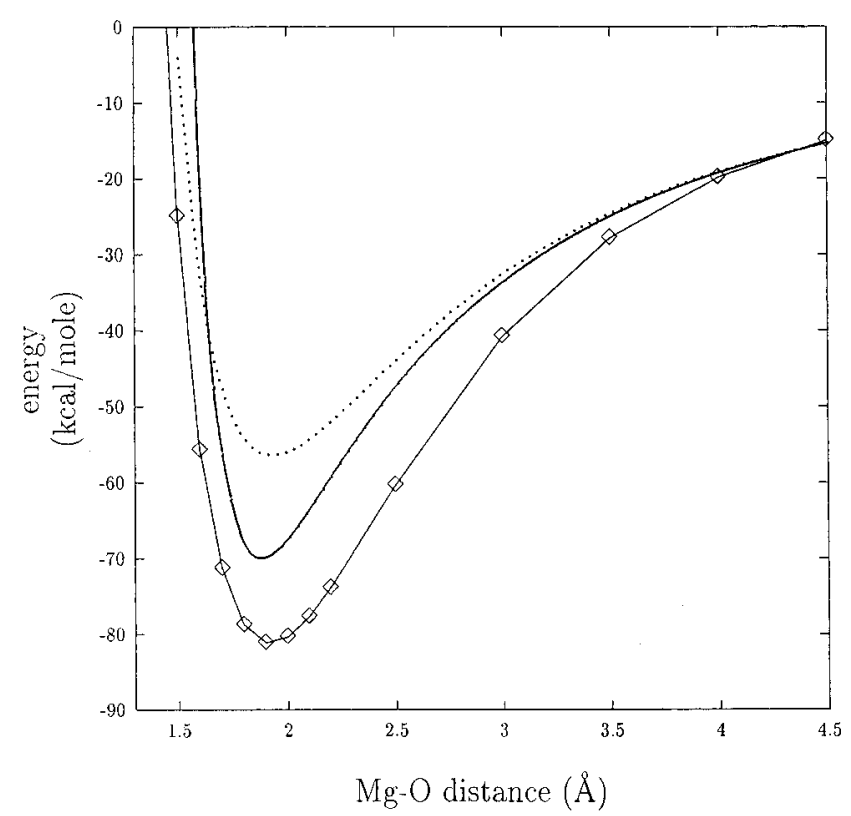

Figure 5. Interaction energy between $\mathrm{Mg}^{2+}$ and a water molecule, as a function of the distance between $\mathrm{Mg}^{2+}$ and the oxygen of the water molecule. $\diamond$ and continuous line: $a b$ initio results. Plain line: effective interaction energy, as obtained with Åqvist's empirical approach. Dotted line: effective interaction energy, as obtained with the present approach, $\mathrm{Mg}^{2+}$ being in the field of five other water molecules.

TABLE 9: $\mathrm{M}^{+}\left(\mathrm{H}_{2} \mathrm{O}\right)_{n}$. Parameters Obtained by Fitting $a b$ Initio Data with 4-Buckingham Functions

\begin{tabular}{llrrc}
\hline cation & $n$ & \multicolumn{1}{c}{$A_{n}$} & \multicolumn{1}{c}{$B_{n}$} & $C_{n}$ \\
\hline $\mathrm{Li}^{+}$ & 1 & 29240.29 & 119.71 & 4.12 \\
$\mathrm{Na}^{+}$ & 1 & 59087.91 & 131.53 & 3.98 \\
$\mathrm{~K}^{+}$ & 1 & 192620.68 & 30.75 & 4.05 \\
$\mathrm{Be}^{2+}$ & 1 & 68896.40 & 856.46 & 4.10 \\
$\mathrm{Mg}^{2+}$ & 1 & 73822.82 & 669.00 & 3.86 \\
$\mathrm{Ca}^{2+}$ & 1 & 76143.74 & 587.88 & 3.55 \\
$\mathrm{Li}^{+}$ & 6 & 42500.40 & -41.73 & 4.87 \\
$\mathrm{Na}^{+}$ & 6 & 61925.75 & 5.53 & 4.13 \\
$\mathrm{~K}^{+}$ & 6 & 296955.35 & -18.84 & 4.24 \\
$\mathrm{Be}^{2+}$ & 4 & 36673.55 & 24.63 & 4.77 \\
$\mathrm{Mg}^{2+}$ & 6 & 129961.00 & -103.79 & 4.81 \\
$\mathrm{Ca}^{2+}$ & 6 & 96027.96 & -25.93 & 3.89
\end{tabular}

TABLE 10: $\mathrm{M}^{+}\left(\mathrm{H}_{2} \mathrm{O}\right)_{n}$. Parameters Obtained by Fitting $a b$ Initio Data with 7-4 Lennard-Jones Functions

\begin{tabular}{llrr}
\hline cation & $n$ & \multicolumn{1}{c}{$A_{n}$} & \multicolumn{1}{c}{$B_{n}$} \\
\hline $\mathrm{Li}^{+}$ & 6 & 568.87 & -12.43 \\
$\mathrm{Na}^{+}$ & 6 & 2825.52 & 105.07 \\
$\mathrm{~K}^{+}$ & 6 & 7234.34 & 167.28 \\
$\mathrm{Be}^{2+}$ & 4 & 562.70 & 49.01 \\
$\mathrm{Mg}^{2+}$ & 6 & 1986.23 & 4.35 \\
$\mathrm{Ca}^{2+}$ & 6 & 6824.99 & 182.48
\end{tabular}

for the data fits. Since our study is aiming at describing atomic interactions as simply as possible, we chose to allow no more than three parameters in the functions, that is, no more than in the Buckingham forms (moreover, developing a new water model lies outside the scope of the present study).

Parameters obtained with the 7-4 Lennard-Jones form are given in Table 10, for the most hydrated systems studied. Here, low msd's were reached with positive $A_{n}$ and $B_{n}$ values, except for $\mathrm{Li}^{+}\left(\mathrm{H}_{2} \mathrm{O}\right)_{6}$. In order to improve data fits, a third parameter was introduced in this form, the charge of the cation, $q_{\mathrm{c}}$, being allowed to differ from +1 or +2 . The corresponding parameters and msd are given in Table 11. Msd values are the lowest found in the present study. Only one large msd remains, for $\mathrm{Be}^{2+}$ $\left(\mathrm{H}_{2} \mathrm{O}\right)_{4}$. Moreover, all parameter values are consistent with their
TABLE 11: $\quad \mathrm{M}^{+}\left(\mathrm{H}_{2} \mathrm{O}\right)_{n}$. Parameters Obtained by Fitting $a b$ Initio Data with 7-4 Lennard-Jones Functions and Adjusted Cation Charges

\begin{tabular}{lccrrc}
\hline cation & $n$ & \multicolumn{1}{c}{$q_{\mathrm{c}}$} & \multicolumn{1}{c}{$A_{n}$} & \multicolumn{1}{c}{$B_{n}$} & $\mathrm{msd}(\mathrm{kcal} / \mathrm{mol})^{2}$ \\
\hline $\mathrm{Li}^{+}$ & 6 & 0.94 & 816.59 & 52.50 & $<0.01$ \\
$\mathrm{Na}^{+}$ & 6 & 0.90 & 3481.47 & 236.86 & 0.01 \\
$\mathrm{~K}^{+}$ & 6 & 0.79 & 11548.95 & 616.93 & $<0.01$ \\
$\mathrm{Be}^{2+}$ & 4 & 1.84 & 758.81 & 158.98 & 0.96 \\
$\mathrm{Mg}^{2+}$ & 6 & 1.74 & 2987.70 & 288.97 & 0.03 \\
$\mathrm{Ca}^{2+}$ & 6 & 1.76 & 8445.10 & 509.43 & 0.07
\end{tabular}

TABLE 12: Radial Distribution Functions of Water Oxygens around Cations as Found in Molecular Dynamics Simulations Performed with 7-4 Lennard-Jones Parameters. Comparison with Experimental Data ${ }^{7}$

\begin{tabular}{lclll}
\hline cation & $R_{\mathrm{h}}^{\text {md }}(\AA)$ & $N_{\mathrm{h}}^{\text {md }}$ & $R_{\mathrm{h}}^{\exp }(\AA)$ & $N_{\mathrm{h}}^{\text {exp }}$ \\
\hline $\mathrm{Li}^{+}$ & $1.90 \pm 0.05$ & 4 & $1.94-2.28$ & $4-6$ \\
$\mathrm{Na}^{+}$ & $2.35 \pm 0.05$ & $5-6$ & $2.40-2.50$ & $4-8$ \\
$\mathrm{~K}^{+}$ & $2.75 \pm 0.05$ & $7-8$ & $2.60-2.95$ & $6-8$ \\
$\mathrm{Be}^{2+}$ & $1.60 \pm 0.05$ & 4 & 1.69 & 4 \\
$\mathrm{Mg}^{2+}$ & $2.05 \pm 0.05$ & 6 & $2.00-2.15$ & 6 \\
$\mathrm{Ca}^{2+}$ & $2.45 \pm 0.05$ & 8 & $2.33-2.49$ & $6-10$
\end{tabular}

expected physical meaning: $A_{n}$ and $B_{n}$ have positive values, and $q_{\mathrm{c}}$ is lower and close to +1 and +2 , for alkaline and alkaline-earth cations, respectively. However, the classical picture corresponding to this parameter set is not satisfactory in one respect: the overall charge of the system is also no more +1 or +2 . As far as cation-water interactions are concerned, this is without any consequence, since charges in the system are effective ones. In particular, during the fitting process, the $q_{\mathrm{c}}$ value helps to correct errors introduced by assuming that atomic charges in the water molecule are those of the TIP3P model. However, if these parameters were to be used in simulations of more complex systems than cations in water solutions, this may lead to artifactual effects. Thus, although the quality of the data fits obtained indicate that this later parameter set deserves further studies, it was not considered in the following.

In order to check that our effective potentials are also able to reproduce $a b$ initio interaction energies of cation-water systems in nonsymmetric configurations, a MD simulation at room temperature of a $\mathrm{Mg}^{2+}\left(\mathrm{H}_{2} \mathrm{O}\right)_{6}$ cluster was performed, from which $25 \mathrm{Mg}^{2+}\left(\mathrm{H}_{2} \mathrm{O}\right)$ geometries were picked. A comparison between $a b$ initio and effective 7-4 Lennard-Jones interaction energies for these geometries is given in Figure 2. The correlation between both is indeed quite strong.

Molecular Dynamics Simulations. The 7-4 Lennard-Jones form was implemented in the CHARMM-22 program package. Parameters given in Table $10\left(q_{\mathrm{c}}=+1\right.$ or +2$)$ were used to compute trajectories of cations in water solutions at room temperature, as detailed in the Methods section. From the last 20 ps of each of these simulations, radial distribution functions of water oxygens around the cation were determined, focusing on $R_{\mathrm{h}}$, the distance corresponding to the first maximum of this function, and on $N_{\mathrm{h}}$, the coordination number, that is, the number of water molecules in the first hydration shell of the cation. $N_{\mathrm{h}}$ and $R_{\mathrm{h}}$ values obtained for each simulation are given in Table 12. They are found to be consistent with experimental data.

For $\mathrm{Na}^{+}$and $\mathrm{K}^{+}$, the first hydration shell is not as well defined as for the other cations, the water molecules of this shell being exchanged with bulk water molecules within the time scale of the simulation. No such water exchange is observed in the alkaline-earth cation simulations. This is consistent with measurements of the unimolecular rate constant of water release from the first hydration shell of small alkaline-earth dications, namely, over $10^{-8} \mathrm{~s}$, while it is well below $10^{-9} \mathrm{~s}$ for alkaline cations. $^{22}$ 
TABLE 13: Differences of Hydration Free Energies of Pairs of Cations Obtained from Perturbation Simulations Performed with 7-4 Lennard-Jones Parameters. Comparison with Experimental Data ${ }^{22,28}$

\begin{tabular}{ccc}
\hline cation a $\rightarrow$ cation b & $\Delta G_{\text {ab }}(\mathrm{kcal} / \mathrm{mol})$ & $\Delta G_{\text {exp }}(\mathrm{kcal} / \mathrm{mol})$ \\
\hline $\mathrm{Li}^{+} \rightarrow \mathrm{Na}^{+}$ & 13.4 & $26.3-27.5$ \\
$\mathrm{Na}^{+} \rightarrow \mathrm{K}^{+}$ & 20.1 & $16.7-17.5$ \\
$\mathrm{Be}^{2+} \rightarrow \mathrm{Mg}^{2+}$ & 98.5 & $120.8-135.0$ \\
$\mathrm{Mg}^{2+} \rightarrow \mathrm{Ca}^{2+}$ & 45.7 & $77.7-80.3$
\end{tabular}

Note that the optimized geometries of the systems studied in order to determine the parameters used in these simulations are also consistent with experimental $R_{\mathrm{h}}$ values. This is not the case when smaller systems ( $n=1$ or $n=2$ cases) are considered (see Tables 1-6). This kind of result supports our approach since it indicates that a system composed of a cation and of the water molecules of its first hydration shell is a good first approximation of a much larger system composed of a cation in a water solution.

Free Energy Difference Calculations. Alkaline cation perturbation simulations were performed with $\mathrm{Na}^{+}$as a starting point, as in Åqvist's work, and alkaline-earth ones with $\mathrm{Mg}^{2+}$ as a starting point, while Åqvist's was $\mathrm{Ca}^{2+}$ (see below). The corresponding differences of hydration free energies are given in Table 13. The errors in the results obtained, as measured by the hysteresis value, ${ }^{2}$ are less than $1 \mathrm{kcal} / \mathrm{mol}$.

Calculated values differ from experimental data by a large amount for $\mathrm{Li}^{+} \rightarrow \mathrm{Na}^{+}, \mathrm{Mg}^{2+} \rightarrow \mathrm{Ca}^{2+}$, and $\mathrm{Be}^{2+} \rightarrow \mathrm{Mg}^{2+}$. In the later case, such a discrepancy was expected since the 7-4 Lennard-Jones form was found to be inaccurate for describing $\mathrm{Be}^{2+}$-water oxygen interactions (see Table 8). In the $\mathrm{Mg}^{2+}$ $\rightarrow \mathrm{Ca}^{2+}$ case, the source of error may lie in the reproduction of the water environment of $\mathrm{Ca}^{2+}$ during $\mathrm{MD}$ simulations. As mentioned in the Introduction, the number of water molecules in the first hydration shell of $\mathrm{Ca}^{2+}$ is a matter of debate, at the experimental as well as at the theoretical level. In particular, neutron diffraction experiments have shown that this number depends upon $\mathrm{Ca}^{2+}$ concentration, namely, that it decreases when the concentration increases. According to these experi-

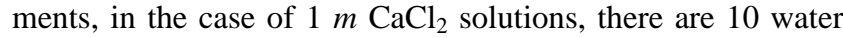
molecules in the first hydration shell of $\mathrm{Ca}^{2+} .23$ Such results are at variance with those obtained from X-ray diffraction experiments, which indicate that there are between 6 and 7 water molecules in the first hydration shell of $\mathrm{Ca}^{2+}$, the former value being the most frequently given one. ${ }^{7}$ Such an experimental controversy suggests that the structure and dynamics of $\mathrm{Ca}^{2+}$ water environment depends on rather subtle physical effects.

In the $\mathrm{Na}^{+} \rightarrow \mathrm{K}^{+}$case, the difference between the free energies of hydration of these two cations is reproduced with a good accuracy, namely, within $2.5-3.5 \mathrm{kcal} / \mathrm{mol}$ of the experimental value. As emphasized hereafter, this later result is likely to be meaningful. First, note that for $\mathrm{Na}^{+}$and $\mathrm{K}^{+}$the fit of $a b$ initio interaction energies was quite accurate (see Table 8). Note also that the interaction energies of these two cations with water molecules are the smallest considered in the present study. This suggests that our approach may be accurate only when the strength of the interactions in the system studied is not too large. The fact that the calculated $\mathrm{Li}^{+} \rightarrow \mathrm{Na}^{+}$free energy difference was not found to be accurate supports this hypothesis. Thus, one may think that, when the interaction strength is important, larger systems need to be considered within the frame of our approach, including at least some water molecules of the second hydration shell.

On the other hand, many-body effects are expected to play a more and more important role as the interaction strength increases. Indeed, as shown in Figures 3-5, for $\mathrm{Na}^{+}$and $\mathrm{K}^{+}$, the difference between their $a b$ initio interaction energy with a single water molecule and the effective interaction energy found in the present work is not large, while it is for $\mathrm{Mg}^{2+}$. However, the striking result is that, for $\mathrm{Na}^{+}$and $\mathrm{K}^{+}$, the effective interactions between these cations and a water molecule in a water solution, as determined by Åvist with his empirical approach or in the present study with an $a b$ initio approach, are very similar (see Figures 3 and 4). Such a result strongly supports Aqvist's approach. Further studies should now be performed in order to check that ab initio calculations performed at higher levels of accuracy (MP2, MP4, etc.) do lead to an even better agreement with experimental data for these two cations, through the parametrization process described in the present study.

For $\mathrm{Mg}^{2+}$, the difference between $a b$ initio and the two effective interaction energies considered underlines the fact that straightforward in vacuo ab initio calculations ( $n=1$ cases) are likely to be not relevant for determining parameters for the corresponding effective potential energy function in solution. On the other hand, the difference between the two kinds of effective interaction energies may come from the fact that Åqvist's method relies on the hypothesis that correct water environments of cations can be obtained during MD simulations. If this hypothesis happens to be not fulfilled in the $\mathrm{Ca}^{2+}$ case, then it is clear that the interaction energy between $\mathrm{Ca}^{2+}$ and one water molecule determined by Áqvist's method is underestimated, if the number of water molecules found in its first hydration shell during the MD simulations is too large, or overestimated, if it is too low. Furthermore, in such cases, the interaction energy between other alkaline-earth cations and one water molecule is underestimated or overestimated as well, since the corresponding parameters were determined so as to reproduce the difference between the hydration free energy of these cations and the hydration free energy of $\mathrm{Ca}^{2+}$. Otherwise, if MD results obtained both by Åqvist and our group happen to be confirmed at the experimental level, that is, if there are indeed eight water molecules in the first hydration shell of $\mathrm{Ca}^{2+}$, this would suggest that studying larger systems in the case of alkaline-earth cations may lead to results closer to those obtained by Åqvist with his empirical approach.

\section{Conclusion}

Starting from $a b$ initio calculations on systems composed of a cation and of the water molecules of its first hydration shell, parameters for the effective interaction between a water molecule and small alkaline and alkaline-earth cations were obtained, using simple water model and potential energy functions. It proved to be possible to reproduce with a good accuracy quantum mechanical interaction energies with simple two-body energy terms, especially as far as alkaline cationwater molecules interactions are concerned. However, when the charge of the cation was allowed to vary during the fitting process, $a b$ initio interactions between $\mathrm{Mg}^{2+}$ or $\mathrm{Ca}^{2+}$ and water molecules were also found to be very well described.

For $\mathrm{Na}^{+}$and $\mathrm{K}^{+}$, the effective interaction obtained was found to be very similar to the effective interaction obtained by $\mathrm{J}$. Åqvist with a purely empirical approach. In this respect, our results support the principles underlying Aqvist's method, namely, seeking for a two-parameter potential energy function able to lead to the reproduction in MD simulations of two experimental values. However, they suggest straightforward improvements. First, a $7-4$, or a $8-4$, Lennard-Jones form should be used in the parameter determination process, instead of the standard 12-6 form. Second, the reference cations for which absolute free energy of hydration are calculated should 
be chosen with great care, since experimental data like hydration numbers may vary a lot. ${ }^{7}$

On a more general level, the present study underlines the fact that radial distribution functions of oxygen atoms around cations are much easier to reproduce than hydration free energy differences, as obtained with perturbation simulations of allatoms classical models. Cations, as in our study $\mathrm{Mg}^{2+}$ and $\mathrm{Be}^{2+}$, may have radial distribution functions in good agreement with experimental data, which are quite homogeneous in these two cases, especially as far as hydration numbers are concerned, ${ }^{7}$ and still have a calculated hydration free energy difference at variance by $20-30 \%$ with the experimental value.

A natural way for improving the results obtained in the present study, and/or generalizing our approach, would be to consider larger systems, namely, systems giving a more representative picture of the environment experienced by a cation in a condensed phase. In line of the present study, such systems should be composed of a cation embedded in two or more hydration shells. Of course, as the size of the system increases, more and more approximations may become necessary, in order to achieve the corresponding ab initio calculations. Thus, our approach will prove wholly successfull only if good effective parameters can be obtained by studying small enough systems. Otherwise, empirical approaches like the one proposed by J. Åqvist will remain necessary for a while.

Acknowledgment. The authors thank J. Parello for fruitful discussions and R. Ahlrichs for kindly providing a version of the TURBOMOLE package.

\section{References and Notes}

(1) Falke, J. J.; Drake, S. K.; Hazard, A. L.; Peersen, O. B. Q. Rev. Biophys. 1995, 27, 219.
(2) Kollman, P. Chem. Rev. 1993, 93, 2395.

(3) Torrie, G. M.; Valleau, J. P. Chem. Phys. Lett. 1974, 28, 578.

(4) Tembe, B. L.; McCammon, J. A. Comput. Chem. 1984, 8, 281

(5) Brooks, B. R.; Bruccoleri, R. E.; Olafson, B. D.; States, D. J.; Swaminathan, S.; Karplus, M. J. Comput. Chem. 1983, 4, 187.

(6) Aqvist, J. J. Phys. Chem. 1990, 94, 8021.

(7) Ohtaki, H.; Radnai, T. Chem. Rev. 1993, 93, 1157. 1588 .

(8) Bernal-Uruchurtu, M. I.; Ortega-Blake, I. J. Chem. Phys. 1995, 103,

(9) Floris, F. M.; Persico, M.; Tani, A.; Tomasi, J. Chem. Phys. Lett. 1994, 227, 126.

(10) Katz, A. Kaufman; Glusker, J. P.; Beebe, S. A.; Bock, C. W. J. Am. Chem. Soc. 1996, 118, 5752.

(11) Ahlrichs, R.; Bar, N.; Haser, M.; Horn, H.; Kolmel, C. Chem. Phys. Lett. 1989, 162, 165.

(12) Ortega-Blake, I.; Barthelat, J. C.; Costes-Puech, E.; Oliveros, E.; Daudey, J. P. J. Chem. Phys. 1982, 76, 4130.

(13) Jorgensen, W. L.; Chandrasekhar, J.; Madura, J. D.; Impey, R. W.; Klein, M. L. J. Chem. Phys. 1983, 79, 926.

(14) Ryckaert, J. P.; Ciccotti, G.; Berendsen, H. J. C. J. Comput. Phys. 1977, 23, 327.

(15) Zwanzig, R. J. Chem. Phys. 1954, 22, 1420.

(16) Bauschlicher, C. W.; Sodupe, M.; Partridge, H. J. Chem. Phys. 1992 96, 4453.

(17) Kaupp, M.; Schleyer, P. R. J. Phys. Chem. 1992, 96, 7316.

(18) Glendening, E. D.; Feller, D. J. Phys. Chem. 1996, 100, 4790.

(19) Bock, C. W.; Katz, A. Kaufman; Glusker, J. P. J. Am. Chem. Soc. 1995, 117, 3754.

(20) Roux, B.; Karplus, M. J. Comput. Chem. 1995, 16, 690.

(21) Kowall, T.; Foglia, F.; Helm, L.; Merbach, A. E. J. Am. Chem. Soc. 1995, 117, 3790.

(22) Marcus, Y. Biophys. Chem. 1994, 51, 111.

(23) Hewish, N. A.; Neilson, G. W.; Enderby, J. E. Nature 1982, 297, 138 .

(24) Krauss, M.; S. W. J.; J. Am. Chem. Soc. 1990, 112, 1460.

(25) Slizys, D.; Marsden, C. J. Private communication, 1996.

(26) Glendening, E. D.; Feller, D. J. Phys. Chem. 1995, 99, 3060

(27) Feller, D.; Glendening, E. D.; Woon, D. E.; Feyereisen, M. W. J. Chem. Phys. 1995, 103, 3526.

(28) Gomer, R.; Tryson, G. J. Chem. Phys. 1977, 66, 4413. 